\title{
Hypotrichosis-deafness syndrome
}

INSERM

\section{Source}

INSERM. (1999). Orphanet: an online rare disease and orphan drug data base.

Hypotrichosis-deafness syndrome. ORPHA:330029

A syndromic genetic deafness characterized by erythrokeratoderma, hypotrichosis, nail dystrophy and sensorineural hearing loss. Erythema, recurrent skin infections and mucositis have also been associated. 